(n)

trobertivier Journal of Nonlinear Mathematical Physics

\title{
On the study of unitary representations of the twisted Heisenberg- Virasoro algebra via highest weight modules over affine Lie algebras*
}

Namhee Kwon

To cite this article: Namhee Kwon (2014) On the study of unitary representations of the twisted Heisenberg-Virasoro algebra via highest weight modules over affine Lie algebras*, Journal of Nonlinear Mathematical Physics 21:4, 584-592, DOI: https://doi.org/10.1080/14029251.2014.975529

To link to this article: https://doi.org/10.1080/14029251.2014.975529

Published online: 04 January 2021 


\title{
On the study of unitary representations of the twisted Heisenberg-Virasoro algebra via highest weight modules over affine Lie algebras*
}

\author{
Namhee Kwon \\ Department of Mathematics, Daegu University, Jillyang, Gyeongsan, \\ Gyeongbuk, 712-714, Republic of Korea \\ nkwon@daegu.ac.kr
}

Received 10 June 2014

Accepted 18 August 2014

\begin{abstract}
In this paper, we first construct an analogue of the Sugawara operators for the twisted Heisenberg-Virasoro algebra. By using these operators, we show that every integrable highest weight module over an affine Lie algebra can be viewed as a unitary representation of the twisted Heisenberg-Virasoro algebra. As a by-product of our constructions, we give the unitary representations of the twisted Heisenberg-Virasoro algebra which have the central charges appearing in [1]. Our approach to obtain these central charges is different with that of [1].
\end{abstract}

Keywords: Twisted Heisenberg-Virasoro algebra; Sugawara operators; affine Lie algebras; unitary representations.

2000 Mathematics Subject Classification: 17B68, 17B65

\section{Introduction}

The twisted Heisenberg-Virasoro algebra $\mathscr{L}$ was first introduced by Arbarello et al. in [1]. This is an infinite dimensional Lie algebra which contains both the Heisenberg algebra and Virasoro algebra as its subalgebras. It is known that the representation theories of both the Virasoro algebra and Heisenberg algebra play important roles in statistical mechanics [2] and string theory [10]. They are also closely related with the theory of affine Lie algebras [8,9].

The representation theory of the twisted Heisenberg-Virasoro algebra $\mathscr{L}$ has been studied by several authors. In particular, in [1] the authors classify the unitary highest weight modules over the twisted Heisenberg-Virasoro algebra.

Let us now briefly describe the results of [1] concerned with the classification of unitary highest weight modules over $\mathscr{L}$.

It is well-known that every unitary highest weight representation of $\mathscr{L}$ can be determined by a quintuple of real numbers $\left(c, h, c_{a}, h_{a}, c_{3}\right)$. (We refer to the appendix of [1] for the details of the real numbers $c, h, c_{a}, h_{a}$ and $c_{3}$.) In [1, (6.6) Theorem], the authors show that every irreducible unitary highest weight representation $R\left(c, h, c_{a}, h_{a}, c_{3}\right)$ of $\mathscr{L}$ is arisen in precisely following cases:

$$
\text { If } c_{a}=0 \text {, then } c_{3}=0, h_{a}=0 \text { and }(c, h)=\left(c_{m}, h_{r, s}^{m}\right) \text {, }
$$

${ }^{*}$ This research was supported by the Daegu University Research Grant, 2013. 
where $c_{m}=1-\frac{6}{(m+2)(m+3)}$ and $h_{r, s}^{m}=\frac{((m+3) r-(m+2) s)^{2}-1}{4(m+2)(m+3)}$ for $m, r, s \in \mathbb{Z}_{\geq 0}$ and $1 \leq s \leq r \leq m+1$.

$$
\begin{aligned}
& \text { If } c_{a}>0, \text { then either }\left\{\begin{array}{l}
c-\frac{12 c_{3}^{2}}{c_{a}} \geq 2 \\
h-\frac{h_{a}^{2}+c_{3}^{2}}{2 c_{a}} \geq 0
\end{array}\right. \\
& \text { or }\left\{\begin{array}{l}
c-\frac{12 c_{3}^{2}}{c_{a}}=1+c_{m} \\
h-\frac{h_{a}^{2}+c_{3}^{2}}{2 c_{a}}=h_{r, s}^{m} .
\end{array}\right.
\end{aligned}
$$

To show the existence of such irreducible unitary representations satisfying the above conditions, in [1] the authors used the tensor product of a highest weight representation of the Heisenberg algebra with a unitary representation of the Virasoro algebra. In their approach, the known classification [2], [3], [4] of unitary highest weight representations of the Virasoro algebra plays an important role.

In this paper, we shall be concerned with a study of unitary representations of $\mathscr{L}$ which does not depend on the classification of unitary highest weight representations of the Virasoro algebra. The key ingredient in our approach is to introduce the twisted Sugawara operators which are variations of the ordinary Sugawara operators. Our construction of the twisted Sugawara operators is based on the construction from string theory which plays an important role in the proofs of $[1,(6.6)$ Theorem, (6.7) Theorem]. By using these new types of the Sugawara operators, we prove that every integrable highest weight representation of an affine Lie algebra yields a unitary representation of the twisted Heisenberg-Virasoro algebra. This generalizes some results of [3] to the twisted Heisenberg-Virasoro algebra (cf. [7, Lecture 10]).

In the last section, we apply our results to the tensor products of the irreducible representations of $\widehat{\mathfrak{s l}_{2}(\mathbb{C})}$. These tensor product representations are equipped with unitarity due to Theorem 3.2. Moreover, our calculations reveal that the conditions for the central charge $c$ appearing in (1.2) and (1.3) can be also obtained through the twisted Sugawara actions on the certain tensor product modules over $\widehat{\mathfrak{s l}_{2}(\mathbb{C})}$.

\section{Preliminaries}

The twisted Heisenberg-Virasoro algebra $\mathscr{L}$ is a complex Lie algebra with a basis $\left\{L_{m}, G_{n}, C, Z, \widetilde{C} \mid m, n \in \mathbb{Z}\right\}$, and its Lie bracket satisfies

$$
\begin{gathered}
{\left[L_{m}, L_{n}\right]=(m-n) L_{m+n}+\frac{m^{3}-m}{12} \delta_{m+n, 0} C .} \\
{\left[G_{m}, G_{n}\right]=m \delta_{m+n, 0} Z .} \\
{\left[G_{m}, L_{n}\right]=m G_{m+n}-i m^{2} \delta_{m+n, 0} \widetilde{C}, \text { where } i \text { is the imaginary unit. }} \\
{[\mathscr{L}, C]=[\mathscr{L}, Z]=[\mathscr{L}, \widetilde{C}]=0 .}
\end{gathered}
$$

We point out that the subalgebras generated by the subsets $\left\{G_{n}, Z \mid n \in \mathbb{Z}\right\}$ and $\left\{L_{j}, C \mid j \in \mathbb{Z}\right\}$ are nothing but the Heisenberg algebra $\mathscr{H}$ and Virasoro algebra Vir, respectively. 
Let us now take a finite dimensional complex simple Lie algebra $\mathfrak{g}$, and fix its Cartan subalgebra $\mathfrak{h}$. Write $\mathfrak{h}_{\mathbb{R}}$ for the real form of $\mathfrak{h}$. We denote by $\tilde{\mathfrak{g}}$ the untwisted affine Lie algebra $\mathbb{C}\left[t, t^{-1}\right] \otimes \mathfrak{g} \oplus$ $\mathbb{C} K$ with commutation relations

$$
\begin{gathered}
{[x(m), y(n)]=[x, y](m+n)+m \delta_{m+n, 0}(x \mid y) K,} \\
{[K, \tilde{\mathfrak{g}}]=0,}
\end{gathered}
$$

where $x(m)$ stands for $t^{m} \otimes x(m \in \mathbb{Z}, x \in \mathfrak{g})$ and $(x \mid y)$ denotes the normalized invariant bilinear form on $\mathfrak{g}$. We also write $\hat{\mathfrak{g}}$ for the affine Lie algebra $\mathbb{C}\left[t, t^{-1}\right] \otimes \mathfrak{g} \oplus \mathbb{C} K \oplus \mathbb{C} d$ which is obtained by adjoining to $\tilde{\mathfrak{g}}$ a derivation $d$. The derivation $d$ satisfies an additional commutation rule $[d, x(n)]=$ $n x(n)$.

A $\tilde{\mathfrak{g}}$-module $V$ is called admissible if for any $v \in V$ we have $x(n) v=0$ for all $x \in \mathfrak{g}$ and all $n>>0$. It is easy to see that all highest weight $\tilde{\mathfrak{g}}$-modules are admissible representations.

In order to perform calculation, it is convenient to introduce the restricted completion $U_{c}(\tilde{\mathfrak{g}})$ of the universal enveloping algebra $U(\tilde{\mathfrak{g}})$. In more details, we define $U_{c}(\tilde{\mathfrak{g}})$ as the set of all series $\sum_{j=1}^{\infty} u_{j}$ with $u_{j} \in U(\tilde{\mathfrak{g}})$ such that for any admissible $\tilde{\mathfrak{g}}$-module $V$ and $v \in V, u_{j}(v)=0$ for all but finitely many $u_{j}$. We identify two series in $U_{c}(\tilde{\mathfrak{g}})$ if they represent the same operator for all admissible $\tilde{\mathfrak{g}}$-modules.

Let $\left\{u_{i}\right\}$ and $\left\{u^{i}\right\}$ be dual bases of $\mathfrak{g}$, i.e. $\left(u_{i} \mid u^{j}\right)=\delta_{i, j}$. Then it is well-known that $\sum_{i} u_{i} u^{i}=$ $\sum_{i} u^{i} u_{i}$, and this yields that

$$
\sum_{i}\left[u_{i}(m), u^{i}(n)\right]=m \delta_{m+n, 0}(\operatorname{dim} \mathfrak{g}) K .
$$

Next, we introduce the Sugawara operators $T_{n}(n \in \mathbb{Z})[6$, Chapter 12] (cf. [3, 4,7]):

$$
\begin{gathered}
T_{0}=\sum_{i} u^{i} u_{i}+2 \sum_{n=1}^{\infty} \sum_{i} u_{i}(-n) u^{i}(n), \\
T_{n}=\sum_{m \in \mathbb{Z}} \sum_{i} u_{i}(-m) u^{i}(m+n) \text { if } n \neq 0 .
\end{gathered}
$$

It is immediate from (2.7) that the Sugawara operators $T_{n}$ are contained in $U_{c}(\tilde{\mathfrak{g}})$.

In a semidirect sum of $U_{c}(\tilde{\mathfrak{g}})$ with $V i r$, we recall that the Lie bracket between the generators of Vir and $U_{c}(\tilde{\mathfrak{g}})$ is given by $\left[x(m), L_{j}\right]=m x(m+j)$ for $x \in \mathfrak{g}$ and $m \in \mathbb{Z}$ (see [5]). This commutation relation is extended to the Sugawara operators as follows.

Lemma 2.1. Let $x \in \mathfrak{g}$ and $m, n \in \mathbb{Z}$. Then, we have

$$
\left[x(m), T_{n}\right]=2(K+g) m x(m+n),
$$

where $g$ denotes the dual Coxeter number of $\mathfrak{g}$.

Proof. See [6, Lemma 12.8]

According to the following theorem, every irreducible highest weight $\tilde{\mathfrak{g}}$-module can be viewed as a representation of Vir. 
Theorem 2.1. Let $L(\Lambda)$ be an irreducible highest weight $\tilde{\mathfrak{g}}$-module with highest weight $\Lambda$. Let $g$ be the dual Coxeter number of $\mathfrak{g}$. Suppose that the level of $\Lambda$ is $k$ and $k \neq-g$. Then, the $\tilde{\mathfrak{g}}-$ module $L(\Lambda)$ is extended to $\tilde{\mathfrak{g}} \rtimes$ Vir-module by letting

$$
L_{n} v=\left(\frac{1}{2(k+g)} T_{n}\right) v \text { for } v \in L(\Lambda) \text { and } n \in \mathbb{Z},
$$

and

$$
C v=\frac{k(\operatorname{dim} \mathfrak{g})}{k+g} v
$$

Proof. This theorem is immediate from both Lemma 2.1 and the following well-known formula

$$
\left[T_{m}, T_{n}\right]=2(K+g)\left((m-n) T_{m+n}+\delta_{m+n, 0} \frac{m^{3}-m}{6}(\operatorname{dimg}) K\right) .
$$

We refer to [7, Theorem 10.1] for the details of (2.8).

Let us now review the coset representation of the Virasoro algebra Vir.

Let $L\left(\Lambda_{1}\right)$ and $L\left(\Lambda_{2}\right)$ be two integrable highest weight representations of $\tilde{\mathfrak{g}}$ with levels $k_{1}$ and $k_{2}$, respectively. Assume that $k_{1}+g \neq 0$ and $k_{2}+g \neq 0$, and write $L_{n}^{\Lambda_{i}}(i=1,2)$ for the action of $L_{n}$ on $L\left(\Lambda_{i}\right)$ to avoid confusion.

For the tensor product $L\left(\Lambda_{1}\right) \otimes L\left(\Lambda_{2}\right)$, we should notice that there are two types of Sugawara actions on $L\left(\Lambda_{1}\right) \otimes L\left(\Lambda_{2}\right)$.

One is obtained by considering the whole tensor product $L\left(\Lambda_{1}\right) \otimes L\left(\Lambda_{2}\right)$ as an admissible $\tilde{\mathfrak{g}}$ module. In this case, the central element $K$ acts as a scalar operator $\left(k_{1}+k_{2}\right) I d$ on $L\left(\Lambda_{1}\right) \otimes L\left(\Lambda_{2}\right)$. So, the corresponding Sugawara operators $L_{n}^{\Lambda_{1} \otimes \Lambda_{2}}$ and $C^{\Lambda_{1} \otimes \Lambda_{2}}$ are given respectively by

$$
L_{n}^{\Lambda_{1} \otimes \Lambda_{2}}=\frac{1}{2\left(k_{1}+k_{2}+g\right)} T_{n}
$$

and

$$
C^{\Lambda_{1} \otimes \Lambda_{2}}=\frac{\left(k_{1}+k_{2}\right) \operatorname{dim} \mathfrak{g}}{k_{1}+k_{2}+g} I d .
$$

The other is obtained through the outer tensor product of representations. This means that the attached Sugawara operators $L_{n}^{\Lambda_{1} \otimes \Lambda_{2}}$ and $C^{\Lambda_{1} \otimes \Lambda_{2}}$ are given respectively by

$$
L_{n}^{\Lambda_{1} \otimes \Lambda_{2}}=L_{n}^{\Lambda_{1}} \otimes 1+1 \otimes L_{n}^{\Lambda_{2}}
$$

and

$$
C^{\Lambda_{1} \otimes \Lambda_{2}}=\left(\frac{k_{1} \operatorname{dimg}}{k_{1}+g}+\frac{k_{2} \operatorname{dim} \mathfrak{g}}{k_{2}+g}\right) I d .
$$

It is known that the map

$$
L_{n} \longrightarrow L_{n}^{\Lambda_{1} \otimes \Lambda_{2}}-L_{n}^{\Lambda_{1} \otimes \Lambda_{2}} \text { and } C \longrightarrow C^{\Lambda_{1} \otimes \Lambda_{2}}-C^{\Lambda_{1} \otimes \Lambda_{2}}
$$

defines a unitary representation of Vir on $L\left(\Lambda_{1}\right) \otimes L\left(\Lambda_{2}\right)$ (see [3], [4], [6, Chapter 12] or [7, Section 10.2]). We call this representation the coset representation of $\operatorname{Vir}$ on $L\left(\Lambda_{1}\right) \otimes L\left(\Lambda_{2}\right)$. 


\section{Twisted Sugawara operators}

In this section, we introduce the twisted Sugawara operators for the twisted Heisenberg-Virasoro algebra $\mathscr{L}$. By using these operators, we extend Theorem 2.1 to the case of the twisted HeisenbergVirasoro algebra. Based on this $\mathscr{L}$-module structure, we also show that every irreducible highest weight module over an affine Lie algebra yields a unitary representation of $\mathscr{L}$.

Theorem 3.1. Let $V$ be an admissible $\tilde{\mathfrak{g}}$-module such that $K$ acts on $V$ as a scalar operator $k I d$, and let $\tilde{c}_{V}$ be any real number attached to the $\tilde{\mathfrak{g}}$-module $V$. Assume that $k \neq 0$ and $k \neq-g$. Suppose that we fix a nonzero element $h_{\mathfrak{g}} \in \mathfrak{h}_{\mathbb{R}}$ such that $\left(h_{\mathfrak{g}} \mid h_{\mathfrak{g}}\right)=1$. Then, the following actions define a representation of $\mathscr{L}$ on $V$ :

$$
\begin{aligned}
L_{0} v & =\left(\frac{1}{2(k+g)} T_{0}+\frac{\tilde{c}_{V}^{2}}{2 k}\right) v \\
L_{n} v & =\left(\frac{1}{2(k+g)} T_{n}+i n \frac{\tilde{c}_{V}}{k} h_{\mathfrak{g}}(n)\right) v \text { for } n \in \mathbb{Z}-\{0\}, \\
G_{n} v & =h_{\mathfrak{g}}(n) v \text { for } n \in \mathbb{Z} \\
Z v & =k v \\
\widetilde{C} v & =\tilde{c}_{V} v \\
C v & =\left(\frac{(\operatorname{dimg}) k}{k+g}+\frac{12 \tilde{c}_{V}^{2}}{k}\right) v
\end{aligned}
$$

where $v \in V$ and $i$ denotes the imaginary unit.

Proof. We show that the actions (3.1) - (3.6) satisfy the commutation rules (2.1) - (2.4).

(a) $\left[L_{m}, L_{n}\right]$ for $m n \neq 0$ and $m+n \neq 0$;

$$
\begin{aligned}
{\left[L_{m}, L_{n}\right] } & =\left[\frac{1}{2(k+g)} T_{m}+i m \frac{\tilde{c}_{V}}{k} h_{\mathfrak{g}}(m), \frac{1}{2(k+g)} T_{n}+i n \frac{\tilde{c}_{V}}{k} h_{\mathfrak{g}}(n)\right] \\
& =\frac{(m-n)}{2(k+g)} T_{m+n}+i \frac{\tilde{c}_{V}}{k} m^{2} h_{\mathfrak{g}}(m+n)-i \frac{\tilde{c}_{V}}{k} n^{2} h_{\mathfrak{g}}(m+n)
\end{aligned}
$$

(by (2.8) and Lemma 2.1)

$$
\begin{aligned}
& =(m-n)\left\{\frac{1}{2(k+g)} T_{m+n}+i \frac{\tilde{c}_{V}}{k}(m+n) h_{\mathfrak{g}}(m+n)\right\} \\
& =(m-n) L_{m+n} .
\end{aligned}
$$

(b) $\left[L_{m}, L_{-m}\right]$ for $m \neq 0$;

$$
\begin{aligned}
& {\left[L_{m}, L_{-m}\right] } \\
= & {\left[\frac{1}{2(k+g)} T_{m}+i m \frac{\tilde{c}_{V}}{k} h_{\mathfrak{g}}(m), \frac{1}{2(k+g)} T_{-m}+i(-m) \frac{\tilde{c}_{V}}{k} h_{\mathfrak{g}}(-m)\right] } \\
= & 2 m\left(\frac{1}{2(k+g)} T_{0}+\frac{\tilde{c}_{V}^{2}}{k} I d\right) v+\frac{m^{3}-m}{12}\left(\frac{12 \tilde{c}_{V}^{2}}{k}+\frac{(\operatorname{dim} \mathfrak{g}) k}{k+g}\right) I d \\
= & 2 m L_{0}+\frac{m^{3}-m}{12} C .
\end{aligned}
$$

(c) $\left[L_{n}, L_{0}\right]$ for $n \in \mathbb{Z}$; 
Namhee Kwon

We may assume that $n \neq 0$. So,

$$
\begin{aligned}
{\left[L_{n}, L_{0}\right] } & =\left[\frac{1}{2(k+g)} T_{n}+i n \frac{\tilde{c}_{V}}{k} h_{\mathfrak{g}}(n), \frac{1}{2(k+g)} T_{0}+\frac{\tilde{c}_{V}^{2}}{2 k}\right] \\
& =n\left(\frac{1}{2(k+g)} T_{n}+i n \frac{\tilde{c}_{V}}{k} h_{\mathfrak{g}}(n)\right) \\
& =n L_{n} .
\end{aligned}
$$

(d) $\left[G_{m}, L_{n}\right]$ for $m, n \in \mathbb{Z}$;

$$
\begin{aligned}
{\left[G_{m}, L_{n}\right] } & =\left[h_{\mathfrak{g}}(m), \frac{1}{2(k+g)} T_{n}+i n \frac{\tilde{c}_{V}}{k} h_{\mathfrak{g}}(n)\right] \\
& =m h_{\mathfrak{g}}(m+n)+i n \frac{\tilde{c}_{V}}{k}\left[h_{\mathfrak{g}}(m), h_{\mathfrak{g}}(n)\right] \\
& = \begin{cases}m h_{\mathfrak{g}}(m+n) & \text { if } n \neq-m \\
m h_{\mathfrak{g}}(0)-i m^{2} \tilde{c}_{V} I d & \text { if } n=-m\end{cases} \\
& =m h_{\mathfrak{g}}(m+n)-i m^{2} \delta_{m+n, 0} \tilde{C}_{V} I d \\
& =m G_{m+n}-i m^{2} \delta_{m+n, 0} \widetilde{C} .
\end{aligned}
$$

Similarly, we can see that $\left[G_{m}, L_{0}\right]=m h_{\mathfrak{g}}(m)=m G_{m}$.

(e) $\left[G_{m}, G_{n}\right]$ for $m, n \in \mathbb{Z}$;

$$
\begin{aligned}
{\left[G_{m}, G_{n}\right] } & =\left[h_{\mathfrak{g}}(m), h_{\mathfrak{g}}(n)\right] \\
& =m \delta_{m+n, 0} k I d \\
& =m \delta_{m+n, 0} Z .
\end{aligned}
$$

Since the central elements $C, Z$ and $\widetilde{C}$ of the algebra $\mathscr{L}$ are scalar operators on $V$, the theorem is now immediate from $(\mathbf{a}),(\mathbf{b}),(\mathbf{c}),(\mathbf{d})$ and $(\mathbf{e})$.

Definition 3.1. We call the operators $\frac{1}{2(k+g)} T_{0}+\frac{\tilde{c}_{V}^{2}}{2 k}$ and $\frac{1}{2(k+g)} T_{n}+i n \frac{\tilde{c}_{V}}{k} h_{\mathfrak{g}}(n)$ in Theorem 3.1 the twisted Sugawara operators associated with the admissible $\tilde{\mathfrak{g}}$-module $V$.

Remark 3.1. We should notice that the twisted Sugawara operators coincide with the ordinary Sugawara operators if $\tilde{c}_{V}=0$ (i.e., the central element $\widetilde{C}$ acts trivially on $V$ ).

Let us now consider an irreducible highest weight $\hat{\mathfrak{g}}$-module $L(\Lambda)$ with highest weight $\Lambda$. We assume that the weight $\Lambda$ is a dominant integral weight and the level of $\Lambda$ is $k$. Then, by Theorem 3.1 the irreducible $\hat{\mathfrak{g}}$-module $L(\Lambda)$ yields a representation of $\mathscr{L}$. In particular, the generator $\widetilde{C}$ acts on $L(\Lambda)$ as $\widetilde{C} v=\tilde{c}_{L(\Lambda)} v$ for some $\tilde{c}_{L(\Lambda)} \in \mathbb{R}$. In this case, we simply write $\tilde{c}_{\Lambda}$ for $\tilde{c}_{L(\Lambda)}$ if no confusion is likely to arise.

Definition 3.2. Let $\mathscr{A}$ be a Lie algebra.

(1) A map $f: \mathscr{A} \longrightarrow \mathscr{A}$ is called a $\overline{\mathbb{C}}$-linear anti-involution of $\mathscr{A}$ if $f(x+y)=f(x)+f(y)$, $f(a x)=\bar{a} f(x), f([x, y])=[f(y), f(x)]$ and $f^{2}=I d$ for all $x, y \in \mathscr{A}$ and $a \in \mathbb{C}$.

(2) A $\mathscr{A}$-module $V$ is unitary if there exists a $\overline{\mathbb{C}}$-linear anti-involution $f$ of $\mathscr{A}$ and a positive definite Hermitian form $\langle$,$\rangle on \mathscr{A}$ which is contravariant with respect to $f$ (i.e., $\langle x u, v\rangle=$ $\langle u, f(x) v\rangle$ for all $u, v \in V$ and $x \in \mathscr{A})$. 
We recall that there uniquely exists a $\overline{\mathbb{C}}$-linear automorphism $\omega_{0}$ of $\mathfrak{g}$ satisfying $\omega_{0}\left(e_{i}\right)=-f_{i}$, $\omega_{0}\left(f_{i}\right)=-e_{i}$ and $\left.\omega_{0}\right|_{\mathfrak{h}_{\mathbb{R}}}=-I d_{\mathfrak{h}_{\mathbb{R}}}$. This automorphism is extended to an involution $\widetilde{\omega}_{0}$ on $\hat{\mathfrak{g}}$ by letting $\widetilde{\omega}_{0}\left(t^{n} \otimes x\right)=t^{-n} \otimes \omega_{0}(x), \widetilde{\omega}_{0}(K)=-K$ and $\widetilde{\omega}_{0}(d)=-d$, where $x \in \mathfrak{g}$. Let $t$ be the transposition map on $\hat{\mathfrak{g}}$ defined as $t(X)=-X$ for $X \in \hat{\mathfrak{g}}$. Then, according to [6, Theorem 11.7] the irreducible $\hat{\mathfrak{g}}-$ module $L(\Lambda)$ carries a unique, up to a constant factor, Hermitian form $\langle$,$\rangle which is nondegenerate,$ positive definite and contravariant with respect to a $\overline{\mathbb{C}}$-linear anti-involution $t \circ \widetilde{\omega}_{0}$.

In the following proposition, we prove that the pairs $\left(L_{n}, L_{-n}\right)$ and $\left(G_{n}, G_{-n}\right)$ are adjoint, and the operators $\widetilde{C}, C$ and $Z$ are self-adjoint.

Proposition 3.1. Let $L(\Lambda)$ be the irreducible highest weight $\hat{\mathfrak{g}}$-module with a dominant integral highest weight $\Lambda$ of level $k$. Let $\langle$,$\rangle be the unique nondegenerate, positive definite, contravariant$ Hermitian form on $L(\Lambda)$ as above. Suppose that $L(\Lambda)$ is equipped with a $\mathscr{L}$-module structure via Theorem 3.1. Then, for $v, w \in L(\Lambda)$ and $n \in \mathbb{Z}$ we have

(1) $\left\langle L_{n} v, w\right\rangle=\left\langle v, L_{-n} w\right\rangle$.

(2) $\left\langle G_{n} v, w\right\rangle=\left\langle v, G_{-n} w\right\rangle$.

(3) $\langle Z v, w\rangle=\langle v, Z w\rangle$.

(4) $\langle\widetilde{C} v, w\rangle=\langle v, \widetilde{C} w\rangle$.

(5) $\langle C v, w\rangle=\langle v, C w\rangle$.

Proof. Since the Hermitian form $\langle$,$\rangle is contravariant with respect to the anti-involution t \circ \widetilde{\omega}_{0}$, we obtain that $\langle X v, w\rangle=-\left\langle v, \widetilde{\omega}_{0}(X) w\right\rangle$ for $v, w \in L(\Lambda)$ and $X \in \hat{\mathfrak{g}}$. Especially, for the fixed element $h_{\mathfrak{g}} \in \mathfrak{h}_{\mathbb{R}}$ we get

$$
\begin{aligned}
\left\langle h_{\mathfrak{g}}(n) v, w\right\rangle & =-\left\langle v, \widetilde{\omega}_{0}\left(h_{\mathfrak{g}}(n)\right) w\right\rangle \\
& =-\left\langle v,-h_{\mathfrak{g}}(-n) w\right\rangle \\
& =\left\langle v, h_{\mathfrak{g}}(-n) w\right\rangle .
\end{aligned}
$$

In addition, we see from [6, Proposition 12.8] that

$$
\left\langle T_{n} v, w\right\rangle=\left\langle v, T_{-n} w\right\rangle \text { for } n \in \mathbb{Z} \text { and } v, w \in L(\Lambda) .
$$

Thus, we have

$$
\begin{aligned}
\left\langle L_{n} v, w\right\rangle= & \left\langle\left(\frac{1}{2(k+g)} T_{n}+i n \frac{\tilde{c}_{\Lambda}}{k} h_{\mathfrak{g}}(n)\right) v, w\right\rangle \\
= & \left\langle v, \frac{1}{2(k+g)} T_{-n} w\right\rangle+\left\langle v,-i n \frac{\tilde{c}_{\Lambda}}{k} h_{\mathfrak{g}}(-n) w\right\rangle \\
& \left(\text { by }(3.9),(3.10) \text { and } \tilde{c}_{\Lambda} \in \mathbb{R}\right) \\
= & \left\langle v,\left(\frac{1}{2(k+g)} T_{-n}-i n \frac{\tilde{c}_{\Lambda}}{k} h_{\mathfrak{g}}(-n)\right) w\right\rangle \\
= & \left\langle v, L_{-n} w\right\rangle .
\end{aligned}
$$

It is also immediate that $\left\langle L_{0} v, w\right\rangle=\left\langle v, L_{0} w\right\rangle$ because $L_{0}=\frac{1}{2(k+g)} T_{0}+\frac{\tilde{c}_{\Lambda}^{2}}{2 k}$ and $\frac{\tilde{c}_{\Lambda}^{2}}{2 k} \in \mathbb{R}$. 
Similarly, we obtain that

$$
\begin{aligned}
\left\langle G_{n} v, w\right\rangle & =\left\langle h_{\mathfrak{g}}(n) v, w\right\rangle \\
& =\left\langle v, h_{\mathfrak{g}}(-n) w\right\rangle \\
& =\left\langle v, G_{-n} w\right\rangle .
\end{aligned}
$$

Finally, since $Z, \widetilde{C}$ and $C$ act on $L(\Lambda)$ as real constant factors, it is clear that $\langle Z v, w\rangle=\langle v, Z w\rangle$, $\langle\widetilde{C} v, w\rangle=\langle v, \widetilde{C} w\rangle$ and $\langle C v, w\rangle=\langle v, C w\rangle$. The result now follows.

Notice that there uniquely exists a $\overline{\mathbb{C}}$-linear anti-involution $\omega$ of $\mathscr{L}$ satisfying $\omega\left(L_{n}\right)=L_{-n}$, $\omega\left(G_{n}\right)=G_{-n}, \omega(Z)=Z, \omega(\widetilde{C})=\widetilde{C}$ and $\omega(C)=C$. Then, the following theorem immediately follows from Theorem 3.1 and Proposition 3.1.

Theorem 3.2. Let $L(\Lambda)$ be the irreducible highest weight $\hat{\mathfrak{g}}$-module with a dominant integral highest weight $\Lambda$. Assume that $L(\Lambda)$ is equipped with a $\mathscr{L}$-module structure via Theorem 3.1. Then, $L(\Lambda)$ is a unitarizable representation of $\mathscr{L}$.

\section{Application to $\widehat{\mathfrak{s l}_{2}(\mathbb{C})}$}

In this section, we apply our results to $\mathfrak{g}=\mathfrak{s l}_{2}(\mathbb{C})$.

By following our notations in Section 2, we have $\tilde{\mathfrak{g}}=\mathbb{C}\left[t, t^{-1}\right] \otimes \mathfrak{s l}_{2}(\mathbb{C}) \oplus \mathbb{C} K$ and $\hat{\mathfrak{g}}=\mathbb{C}\left[t, t^{-1}\right] \otimes$ $\mathfrak{s l}_{2}(\mathbb{C}) \oplus \mathbb{C} K \oplus \mathbb{C} d$. Let $\mathfrak{h}$ be the Cartan subalgebra $\mathbb{C}\left(\begin{array}{cc}1 & 0 \\ 0 & -1\end{array}\right)$ of $\mathfrak{g}$, and let $\alpha: \mathfrak{h} \longrightarrow \mathbb{C}$ be the simple root defined as $\alpha\left(\begin{array}{cc}h & 0 \\ 0 & -h\end{array}\right)=2 h$. We introduce a nondegenerate symmetric bilinear form $(\mid)$ on $\hat{\mathfrak{h}}=$ $1 \otimes \mathfrak{h} \oplus \mathbb{C} K \oplus \mathbb{C} d$ satisfying $(\alpha \mid \alpha)=2,(K \mid d)=1$ and $(\alpha \mid K)=(\alpha \mid d)=(d \mid d)=(K \mid K)=0$ through identification $\hat{\mathfrak{h}} \simeq \hat{\mathfrak{h}}^{*}$.

Set $P_{+}=\left\{\lambda \in \hat{\mathfrak{h}}^{*} \mid \frac{2(\lambda \mid \alpha)}{(\alpha \mid \alpha)} \in \mathbb{Z}_{\geq 0}\right.$ and $\left.\frac{2(\lambda \mid K-\alpha)}{(K-\alpha \mid K-\alpha)} \in \mathbb{Z}_{\geq 0}\right\}$. Then, by simple computations we get $P_{+}=\left\{\frac{1}{2} n \alpha+m d+b K \mid m, n \in \mathbb{Z}_{\geq 0}, n \leq m, b \in \mathbb{C}\right\}$.

Choose two integral dominant weights $\lambda=\frac{1}{2} n \alpha+m d\left(m \in \mathbb{Z}_{\geq 1}\right)$ and $d$ in $P_{+}$, and consider the corresponding integrable highest weight $\hat{\mathfrak{g}}$-modules $L(\lambda)$ and $L(d)$. According to [6, Lemma 9.10], $L(\lambda)$ and $L(d)$ are still integrable highest weight $\tilde{\mathfrak{g}}$-modules and the central element $K$ acts on $L(\lambda)$ and $L(d)$ as $m I d$ and $I d$, respectively. Also, by Theorem 3.1 the representations $L(\lambda)$ and $L(d)$ are equipped with $\mathscr{L}$-module structures, and we take $\tilde{c}_{\lambda}$ as any real number and $\tilde{c}_{d}=0$ for our purpose. By tensoring $L(\lambda)$ with $L(d)$, we obtain a tensor product representation $L(\lambda) \otimes L(d)$ of $\mathscr{L}$. We point out that the tensor product $L(\lambda) \otimes L(d)$ is a unitary representation of $\mathscr{L}$ due to Theorem 3.2. In addition, the central elements $\widetilde{C}$ and $Z$ act on $L(\lambda) \otimes L(d)$ as $\tilde{c}_{\lambda} I d$ and $(m+1) I d$, respectively. Let $c$ be the eigenvalue of the central element $C$ on $L(\lambda) \otimes L(d)$. Then, by Equation (3.6) we get that $c=\frac{3 m}{m+2}+\frac{12 \tilde{c}_{\lambda}^{2}}{m}+1$. So, we have $c-\frac{12 \tilde{c}_{\lambda}^{2}}{m}=4-\frac{6}{m+2}$ for $m \in \mathbb{Z}_{\geq 1}$. Hence, this implies that $c-\frac{12 \tilde{c}_{\lambda}^{2}}{m+1} \geq 2$ (cf. [1, (6.6) Theorem] or Equation (1.2)).

Next, let us endow $L(\lambda) \otimes L(d)$ with the coset Vir-module structure (see Section 2 for the details of the coset Virasoro actions). Then, we can trivially extend this coset Vir-module structure to a $\mathscr{L}$-module structure on $L(\lambda) \otimes L(d)$ as follows:

(1) the generators $L_{n}$ and $C$ act on $L(\lambda) \otimes L(d)$ through the coset Virasoro actions defined in (2.9). 
(2) the generators $G_{n}, Z$, and $\widetilde{C}$ act trivially on $L(\lambda) \otimes L(d)$.

We should notice that $L(\lambda) \otimes L(d)$ becomes a unitary representation of $\mathscr{L}$ under the actions (1) and (2). We also see from (2.9) that the element $C$ acts on $L(\lambda) \otimes L(d)$ as $\left(\frac{3 m}{m+2}+1-\frac{3(m+1)}{m+3}\right) I d$. Let us now take the tensor product of $L(\lambda) \otimes L(d)$ with the integrable highest weight $\tilde{\mathfrak{g}}$-module $L(d+\alpha)$. Then, we obtain a unitary representation $(L(\lambda) \otimes L(d)) \otimes L(d+\alpha)$ of $\mathscr{L}$. (Here, $L(d+\alpha)$ is considered as a unitary representation of $\mathscr{L}$ through Theorem 3.2.) Note that the central elements $\widetilde{C}$ and $Z$ act on $(L(\lambda) \otimes L(d)) \otimes L(d+\alpha)$ as $\tilde{c}_{d+\alpha} I d$ and $I d$, respectively. In addition, the eigenvalue $c$ of the central element $C$ on $(L(\lambda) \otimes L(d)) \otimes L(d+\alpha)$ is

$$
\left(\frac{3 m}{m+2}+1-\frac{3(m+1)}{m+3}\right)+\left(1+12 \tilde{c}_{d+\alpha}^{2}\right) .
$$

Thus, these yield that $c-12 \tilde{c}_{d+\alpha}^{2}=2-\frac{6}{(m+2)(m+3)}$ which is a special case of Equation (1.3) when $c_{a}=1$.

\section{References}

[1] E. Arbarello, C. De Concini, V. G. Kac and C. Procesi, Moduli spaces of curves and representation theory, Comm. Math. Phys. 117 (1988) 1-36.

[2] D. Friedan, Z. Qiu and S. Shenker, Conformal invariance, Unitarity and critical exponents in two dimensions, Phys. Rev. Lett. 52 (1984) 1575-1578.

[3] P. Goddard, A. Kent and D. Olive, Virasoro algebras and coset space modules, Phys. Lett. B 152 (1985) $88-92$.

[4] P. Goddard, A. Kent and D. Olive, Unitary representations of the Virasoro and super-Virasoro algebras, Comm. Math. Phys 103 (1986) 105-119.

[5] V. G. Kac, Highest weight representations of conformal current algebras, in Symposium on topological and geometrical methods in field theory, (World Scientific, Espoo, 1986), pp. 3-16.

[6] V. G. Kac, Infinite dimensional Lie algebras (Cambridge Univ. Press, Cambridge, 1990).

[7] V. G. Kac and A. K. Raina, Bombay lectures on highest weight representations of infinite-dimensional Lie algebras, Adv. Ser. Math. Phys. 2 (World Scientific, Singapore, New Jersey, Hong Kong 1987).

[8] V. G. Kac and M. Wakimoto, Unitarizable highest weight representation of the Virasoro, NeveuSchwarz and Ramond algebras, Conformal groups and related symmetries physical results and mathematical background, Lect. Notes in Phys 261 (Springer-Verlag, 1986) 345-372.

[9] V. G. Kac and M. Wakimoto, Modular and conformal invariance constraints in representation theory of affine algebras, Adv. Math 70 (1988) 156-236.

[10] J. H. Schwarz, Superstring theory, Physics Rep. 83 (1982), 223-322. 